SHORT REPORT

\title{
Older people's understanding of cumulative risks when provided with annual stroke risk information
}

\section{R Fuller, N Dudley, J Blacktop}

See end of article for

authors' affiliations

.....................

Correspondence to:

Dr Richard Fuller, Medical

Department for the Elderly,

General Infirmary at Leeds,

Leeds LSI 3EX, UK;

Ftworf@aol.com

Submitted 21 January 2004

Accepted

15 February 2004
Postgrad Med J 2004;80:677-678. doi: 10.1136/pgmj.2004.019489

\begin{abstract}
Understanding how cumulative health risks increase over time constitutes an important part of decision making about treatment risks and benefits. In this study, wide ranging perceptions of cumulative risk led to significant overestimation and underestimation of risks over time. Time has shown to significantly impact on the personal interpretation of cumulative risk information by patients, with many subjects struggling to understand the concept of cumulative risks. This suggests that a careful explanation of the effect of cumulative risk over time to patients should contribute to more effective decision making.
\end{abstract}

A $\mathrm{n}$ increasing focus is placed upon effective risk communication between doctors and patients, highlighting the expert role of patients and the need for informed choice. ${ }^{2}$ Patients have difficulties understanding risk information, and this is not helped by the way such information is presented, or the potential impact of framing effects on decisions. ${ }^{3}$ Comparisons with everyday risk and the use of pictorial information can aid understanding and successful risk communication. ${ }^{4}$

While some risks relate to fixed episodes in time-for example, the risk of perioperative death-other diseases may carry cumulative risks. An example of this is the risk of stroke associated with atrial fibrillation. Discussing this sort of cumulative risk information may pose additional challenges for both doctors and patients, as the extent to which patients are able to interpret this cumulative data and use it in decision making is unknown.

This study aimed to investigate patients' understanding of cumulative risk when presented with information relating to atrial fibrillation and annual stroke risks.

\section{PATIENTS, METHODS, AND RESULTS}

A total of 285 patients attending an elderly medicine clinic at St James's Hospital were assessed by one of the researchers (RF) for entry into this study, excluding those with significant audiovisual (speech, sight, or hearing impairment affecting reading or communication ability) or cognitive impairment (Abbreviated Mental Test Score <8/10). Participants answered a questionnaire administered by a researcher based on stroke prevention treatments in a scenario of atrial fibrillation and a recent ischaemic stroke. Two treatments were offered, one carrying an annual risk of stroke of $4 \%$ and the other $12 \%$, reflecting the risk reduction of anticoagulants over placebo. ${ }^{5}$

Older people can understand pictorial representations of probability, and a crowd figure pictogram was used in this study to demonstrate the risks of stroke with both treatments. This pictogram can be found in a previous study where it has been used successfully to aid probability understanding by older people. ${ }^{6}$ Participants were then asked to mark out a proportion of figures based on the number of people they felt would have had a stroke after five and 10 years for both treatments.
The study was discussed with 102 eligible patients, and 81 consented to participate: 45 male, 36 female, age range 66-97 years (median 81 years).

The range of answers given by participants demonstrated a wide misunderstanding of cumulative probability information. The majority of respondents perceived cumulative probabilities as intuitive multiplications of the risk (for example, $4 \%$ at five years $=20 \%$ ), with incorrect estimations of the true risk. When asked to judge the number of people suffering a stroke after 10 years on the treatment bearing an annual risk of $12 \%$, many respondents marked out the entire pictogram of 100 figures, an overestimation of almost 30\%.

Responses for all questions were distributed across a range of answers with significant underestimation and overestimations of cumulative probability being expressed, with an annual $4 \%$ risk at five years being interpreted anywhere from $2 \%$ to $100 \%$ (fig 1 ). Other respondents' answers showed a decrease in cumulative probability as time increased, with a $12 \%$ annual risk over 10 years marked as less than $20 \%$ by 10 (14\%) respondents (fig 2). Many struggled with understanding this form of risk information, with up to 13 (16\%) unable to answer the questions.

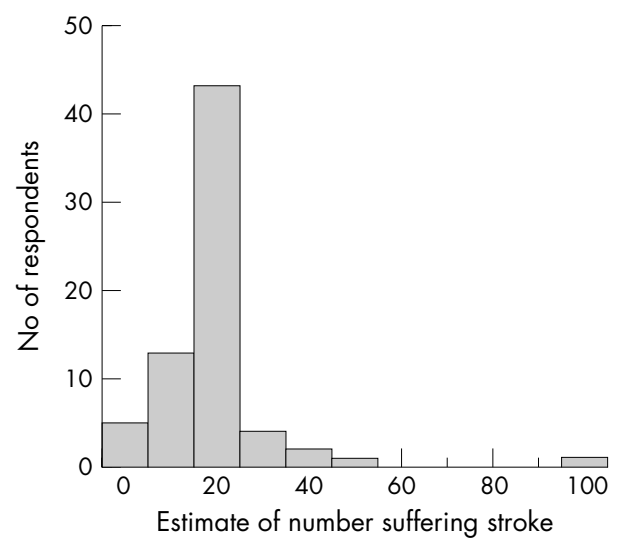

Figure 1 Cumulative estimates of annual $4 \%$ risk at five years. 


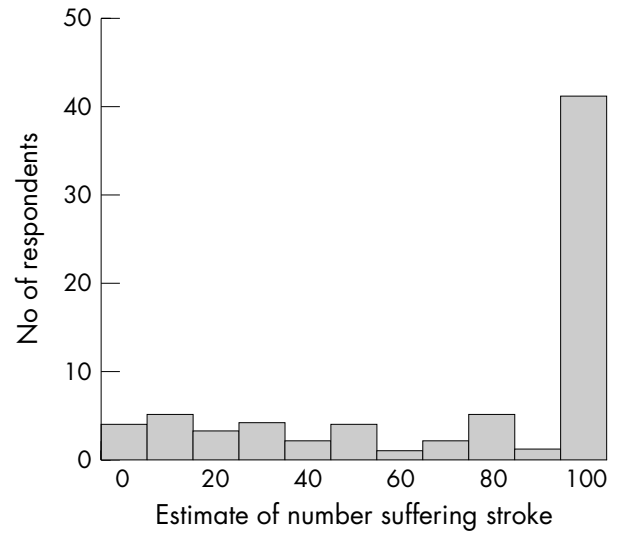

Figure 2 Cumulative estimates of annual $12 \%$ risk at 10 years.

\section{DISCUSSION}

The extent to which cumulative data is presented to, and interpreted by, patients is rarely highlighted, and this study has shown wide reaching misunderstanding of cumulative risk information. Many respondents intuitively multiplied up cumulative probabilities, leading to significant overestimations in risk over time. Such misperceptions may lead to patients declining treatments or over-rating treatment benefits, as demonstrated in patients' perception of risk information before carotid endarterectomy surgery. ${ }^{7}$

Understanding information about the risks and benefits of treatment is a key factor in individual patients' contributions to decision making. Correct interpretation of cumulative probability information is equally important, as differences between treatments after one year may not appear so obvious. However over a longer time span these differences are likely to be more apparent to patients, impacting on their decisions. The difference between placebo and warfarin in preventing strokes in atrial fibrillation demonstrates this well: a stroke risk of $4 \% \vee 12 \%$ after one year, but a risk of $34 \% \vee 72 \%$ at 10 years.

However, it is unlikely that the responses seen just reflect simple misinterpretation of probabilities. Patients' perceptions of the personal effects of risk heavily influence errors in risk information, as the "distancing" of health risks leads to their underestimation. ${ }^{8}$ In the carotid surgery trial, patients' perceptions of the risks and benefits of surgery rose as the operation date neared, ${ }^{7}$ and similar overestimations may have occurred in this study in relation to personal experience of stroke. We accept that this study did not investigate the impact of elders' perceptions of their own longevity, views that are likely to impact on individuals' health beliefs. ${ }^{9}$ This remains a largely unresearched area, and it would be of interest to examine the views of elders about longevity when discussing cumulative risk information and the consequent effect on decision making.

Discussing risks and benefits should not represent a simple exchange of numerical information between clinicians and patients, irrespective of presentation. This study has shown that time has a significant impact on personal interpretation
Learning points

- Effective risk communication forms a cornerstone of good medical practice.

- Many diseases and treatments carry cumulative risks and benefits (for example, stroke prevention and anticoagulation). Understanding how these risks increase over time influences patient decision making.

- Significant misinterpretation of cumulative risks was demonstrated in this study, with underestimation and overestimation through "distancing" or overperception of cumulative risks. This may in part explain the low uptake of longer term treatments such as anticoagulation for stroke prevention.

- To maximise patients' abilities to make decisions based on longer term risk and benefits, cumulative risk information should be presented by comparisons over time rather than relying on patients' interpretation of annual risk rates.

of risk information by patients, and may begin to explain some of the apparent underuse of long term treatments such as anticoagulation in stroke prevention. ${ }^{10}$ In order to maximise patients' ability to make informed decisions regarding longer term risks and benefits of treatment, we would recommend that such information is carefully presented by comparisons at, for example, one, five, and 10 years as appropriate.

\section{Authors' affiliations}

R Fuller, Medical Department for the Elderly, General Infirmary at Leeds, Leeds, UK

N Dudley, Elderly Services, St James's University Hospital, Leeds, UK J Blacktop, School of Human and Health Sciences, University of Huddersfield, Huddersfield, UK

\section{REFERENCES}

1 Mazur DJ. Influence of the law on risk and informed consent. BMJ 2003;327:731-6.

2 Department of Health. The expert patient: a new approach to chronic disease management for the 21 st century. London: DoH, 2001.

3 Thornton $\mathbf{H}$. Patients' understanding of risk: enabling understanding must not lead to manipulation. BMJ 2003;327:693-4.

4 Edwards AGK, Elwyn GJ, Mulley A. Explaining risks: turning numerical data into meaningful pictures. BMJ 2002;324:827-830.

5 Hart RG. Warfarin in atrial fibrillation: underused in the elderly, often inappropriately used in the young. Heart 1999;82:539-40.

6 Fuller R, Dudley NJ, Blacktop J. How informed is consent? Understanding of verbal and pictorial probability information by medical inpatients. Postgrad Med J 2002;78:543-4.

7 Lloyd A, Hayes PR, Naylor AR. The role of risk and perception in informed consent for surgery. Medical Decision Making 2001;21:141-9.

8 Frewer $\mathrm{L}$, Howard $\mathrm{C}$, Hedderly $\mathrm{D}$, et al. The use of the elaboration model in developing effective food risk communication. Risk Analysis 1997; 17:269-81.

9 Idler EL, Kasl S. Health perceptions and survival: do global evaluations of health status really predict mortality? J Gerontol 1991;46:S55-65.

10 Sudlow M, Thomson R, Thwaites B, et al. Prevalence of atrial fibrillation and eligibility for anticoagulants in the community. Lancet 1998;352:1167-71. 